\title{
Function Design of the Hydraulic Manifold Block
}

\author{
Yin Sai Guo ${ }^{1, a^{*}}$, Yi Zhang ${ }^{1, b}$ \\ ${ }^{1}$ School of Mechanical Engineering, Xijing University, Xi'an710123, China; \\ $\mathrm{a}^{*}$ guoysxj@163.com, b'zhangyjd@163.com,
}

Keywords: Hydraulic manifold block, valve block, virtual design, function design.

\begin{abstract}
Hydraulic integrated block design is a creative work. In addition to the needed calculation for the design and checking of the hole, a lot of the content of the work belongs to the non-numerical calculation. The wisdom and creative of the designers are used, at the same time the computer-aided design technology is used to explore the design method of hydraulic manifold block. The virtual design is used to realize function design of hydraulic manifold block. The function design mainly includes digitization of the hydraulic principle diagram, the design of the valve plate, the hole connecting design of hydraulic manifold block and internal hole checking of the hydraulic manifold block.
\end{abstract}

\section{Introduction}

Hydraulic manifold block (HMB) is the valve block drilled in many holes [1]. Its external is equipped with all kinds of Hydraulic components, such as hydraulic valve, pipe joint, pressure gauge, etc [2]. Its internal hole and element channel are interlinked. The hydraulic integrated circuits are made to realize the system control requirements [3] [4]. The hydraulic system using the integrated block has the following four characteristics. (1) Reduce the labor of valve and pipeline installation and the existing equipment is upgraded by using the technology. (2) Reduce the size and the weight of the hydraulic system to achieve more function in the small space near the actuator. (3) Reduce installation of exposure pipeline and pipeline connection to lower the possibility of leakage. (4) Without destroying the outside line connection, quickly remove and replace the components to improve the system suitability. Hydraulic manifold block has the advantages of a compact structure, easy installation and maintenance, less leakage, a small area, easily realizing standardization, which is widely used in all kinds of hydraulic system, as is showed in figure 1 and 2.

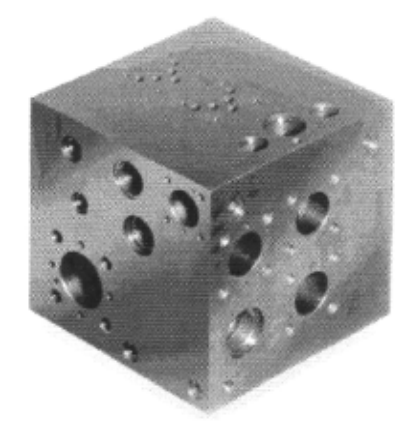

Fig. 1 Hydraulic manifold block

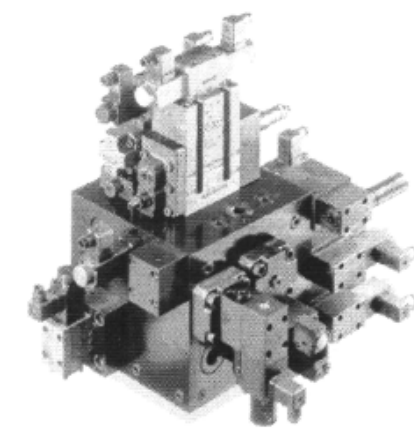

Fig. 2 Hydraulic integrated block assembly

\section{Digitization of the Hydraulic Principle Diagram}

According to the hydraulic principle diagram, the schematic diagram connecting information of the integrated part is formed which provides the basis for a valve plate design, hole connecting design and hole checking. Hydraulic integrated block diagram is described, whose propose is to get the type and information of the integrated block valve, the connecting relations of the oil mouth piece between valve and valve, and the connecting relations of the public oil and mouth valve port. 


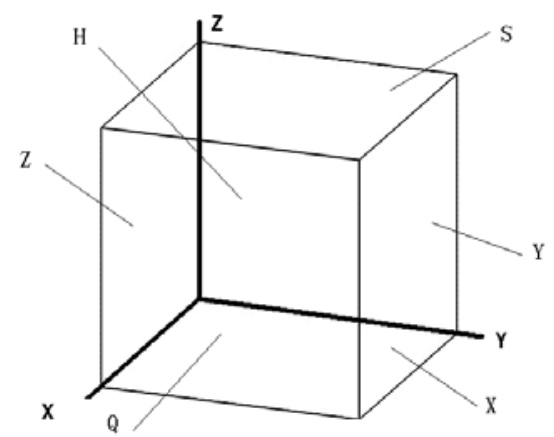

Fig. 3 Coordinate system established

As the design and connectivity of each hole of hydraulic manifold block and the valve installation have the specific location coordinates, and specific location coordinates of different coordinates are not the same, a unified coordinate system is established. The hydraulic manifold block behind the lower left corner is set as the origin of coordinates. The front, back, left, right, up and down of six surfaces respectively are defined as Q, H, Z, X, Y and S. The establishment of coordinate system is shown in figure 3.

\section{Design of the Valve Plate}

The design of the valve plate refers to reasonable placement of the hydraulic components on the hydraulic manifold block solve the hydraulic element space interference and provide reliable and reasonable space position parameters of the hydraulic element for holes connecting design [5].

Hydraulic components on the hydraulic manifold block placement principles:

(1) Valve block geometry size should concern the overall dimensions of each element installed on the valve block which makes each element has enough assembly space.

(2) Element layout should follow the principle of priority and the element layout priority is the order of element layout.

(3) In the layout, the installation direction reasonability of the valve body should be considered. The direction of the valve core is in the horizontal direction to prevent the sensitivity from effect of the valve weight.

(4) The layout of the hydraulic components on the hydraulic manifold block takes the minimal machining hole as principle.

(5) The strength checking of the minimum wall thickness h between impassability holes must be conducted, as is shown in figure 4.

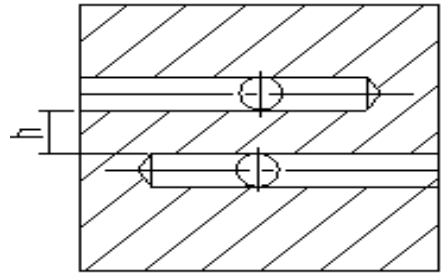

Fig. 4 Minimum wall thickness h between impassability holes

\section{Hole Connecting Design of Hydraulic Manifold Block}

When the connecting relation, pore diameter, position, depth and surface number of holes are established, automatic connecting of holes is implemented. Set respectively length, width and height of integrated block as l, d, h. Set respectively front, back, left, right, above and below of integrated block as Q, H, Y, Z, S, X, respectively. X, y, z represent respectively the $\mathrm{x}, \mathrm{y}, \mathrm{z}$ direction coordinates of the hole. R, dep, gy, represent respectively hole radius, hole depth and auxiliary hole.

For example, 1 hole on the front (Q) and 2 hole on the left (Z): 


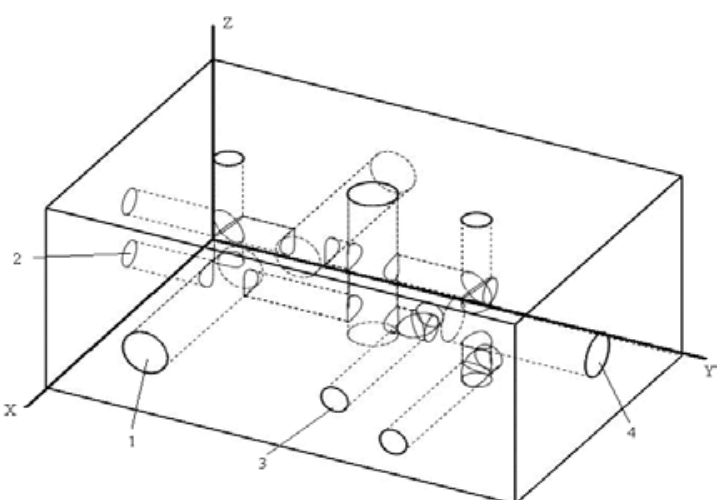

Fig. 5 Connection of hole 1 and 2

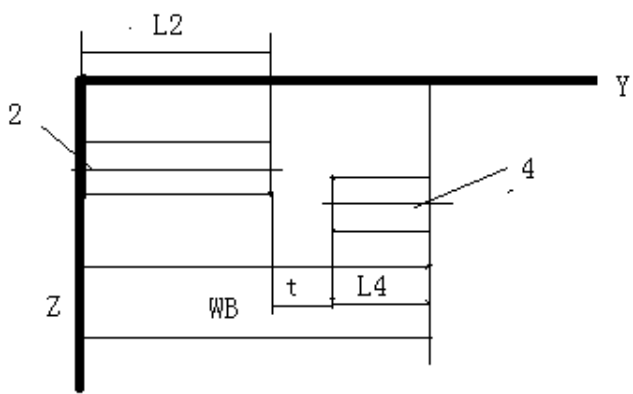

Fig. 6 Two holes location at opposite surface

1. When $\mid \mathrm{z} 2$ - z1 $\mid<\mathrm{r} 1+\mathrm{r} 2$, two holes can be directly connected, dep1 = d - x2, dep2 = y1, as is shown in figure 5 .

2. When $\mid \mathrm{z} 2$ - z1 $\mid \geq \mathrm{r} 1+\mathrm{r} 2$, it can be divided into four kinds of case discussion:

(1) When z2 > z1, z1 > h / 2, dep1 = d - x2, dep2 = y1, auxiliary hole should be drilled on the top (S).

Coordinates of auxiliary hole: $x g y=x 2$, ygy $=y 1$;

Depth of auxiliary hole: depgy=h-z1.

(2) When $\mathrm{z} 2>\mathrm{z} 1, \mathrm{z} 2 \mathrm{~h} / \mathrm{2}$, dep1 $=\mathrm{d}-\mathrm{x} 2$, dep2 = y1, auxiliary hole should be drilled on the below (X).

Coordinates of auxiliary hole: $x g y=x 2$, $y g y=y 1$;

Depth of auxiliary hole: dep gy=z1.

(3) When $z 2 \leq z 1, z 2>h / 2$, dep1=d-x2,dep2=y2, auxiliary hole should be drilled on the above (S).

Coordinates of auxiliary hole: $x g y=x 2$, $y g y=y 1$;

Depth of auxiliary hole: dep gy=h-z2.

(4) When $\mathrm{z} 2<\mathrm{z} 1, \mathrm{z} 1<\mathrm{h} / 2$, dep1=d-x2, dep2=y2, auxiliary hole should be drilled on the below (X).

Coordinates of auxiliary hole: $x g y=x 2$, ygy $=y 1$;

Depth of auxiliary hole: dep gy $=\mathrm{h}-\mathrm{z} 2$.

Similarly, the design and calculation are carried on holes on the other two adjacent sides. According to the above holes design, the hole list and hole connected tables are established.

\section{Internal Hole Checking of the Hydraulic Manifold Block}

According to the valve block hole design results, hydraulic manifold block is checked to determine whether the position relationship between each hole and all other holes of the valve block meet the requirements of the design of the system or not. According to two holes construction base, the dangerous hole of internal hole of hydraulic manifold blocks is judged, which is divided into the following two kinds of circumstances: (1) Two holes at the same surface; (2) Two holes at the opposite surface.

(1) Two holes at the same surface.

The minimum wall thickness between the two holes from figure 5:

$W_{\min }=\sqrt{(y 1-y 3)^{2}+(z 1-z 3)^{2}}-(\mathrm{R} 1+\mathrm{R} 3)$

If $\mathrm{W}_{\min }<0$, the two holes are connection.

If $0 \leq \mathrm{W}_{\min }<\mathrm{WALL}$, the two holes are not connection but did not meet the requirements of security wall thickness.

If $\mathrm{W}_{\text {min }} \geq \mathrm{WALL}$, the two holes are not connection and meet the requirements of security wall thickness.

(2) Two holes at the opposite surface.

The formula is got from Figure 5 and 6:

$\mathrm{t}=\mathrm{WB}-(\mathrm{L} 2+\mathrm{L} 4), \quad W=\sqrt{(y 2-y 4)^{2}+(\mathrm{z} 2-\mathrm{z} 4)^{2}}-(\mathrm{R} 1+\mathrm{R} 3)$ 
When $\mathrm{t} \geq \mathrm{WALL} \cup \mathrm{W} \geq \mathrm{WALL}$, the two holes are not connection and meet the requirements of security wall thickness.

When $\mathrm{t} \leq 0 \cap \mathrm{W}<\mathrm{WALL}$,

If $0 \leq \mathrm{W}<\mathrm{WALL}$, the two holes are not connection but did not meet the requirements of security wall thickness.

If $\mathrm{W}<0$, the two holes are connection.

\section{Summary}

The virtual design is used to realize function design of hydraulic manifold block. The function design mainly includes digitization of the hydraulic principle diagram, the design of the valve plate, the hole connecting design of hydraulic manifold block and internal hole checking of the hydraulic manifold block. It provides an efficient and practical new method for the function design of hydraulic manifold block.

\section{Acknowledgements}

This study was supported by the Natural Science Foundation of Shaanxi Province (Program No. 2013JM8040), the Scientific Research Program Funded by Shaanxi Provincial Education Department (Program No. 2013JK1204) and Graduate Student Innovation Fund of Xijing University.

\section{References}

[1]Wei-Min L I, Xie L Y. On the SolidWorks-Based Virtual Design Integrated Hydraulic Manifold Blocks[J]. Journal of Northeastern University, 2006, 27(6):681-684.

[2]Feng X U. Study on the Design of Hydraulic Manifold Block Based on Pro/E[J]. Journal of Nanjing Institute of Industry Technology, 2004.

[3]Liu H P, Wei-Min L I, Cui J W. Virtual Design of Hydraulic Manifold Block[J]. Journal of Liaoning Institute of Technology, 2007.

[4]FENG Yi (, ), Zhang H, et al. Design of hydraulic manifold blocks based on intelligent virtual design method[J]. Computer Integrated Manufacturing Systems, 2006, 12(3):339-345.

[5]Jin Y. Design of Hydraulic Fault Diagnosis System Based on Virtual Instrument[J]. Microcomputer Information, 2010, 26(4):113-115. 